\title{
The Spectrum Power of Interstellar Plasma Inhomogeneities in the Direction of Eleven Radio Pulsars
}

\author{
M. V. Popov ${ }^{a}, *$ and T. V. Smirnova ${ }^{b, * *}$ \\ ${ }^{a}$ Astro-Space Center of the P.N. Lebedev Physical Institute of RAS, Moscow, Russia \\ ${ }^{b}$ Puschino Radio Astronomical Observatory of the Astro-Space Center \\ of the P.N. Lebedev Physical Institute of RAS, Puschino, Russia \\ *e-mail:popov069@asc.rssi.ru \\ **e-mail:tania@prao.ru \\ Received July 21, 2021; revised August 15, 2021; accepted August 31, 2021
}

\begin{abstract}
We have analyzed two-dimensional correlation functions from the dynamic spectra of 11 pulsars using the archival data of the "Radioastron" project. The time-sections of these functions were approximated by exponential functions with a power $\alpha$. It is shown that this approximation describes the shape of the correlation function much better than the Gaussian. The temporal structure function $D(\Delta t)$ for small values of the delay $\Delta t$ is a power law with an index $\alpha$. The spectrum power of spatial inhomogeneities of the interstellar plasma is related to the power of the structure function as $n=\alpha+2$. We have determined the characteristic scintillation time and the power $n$ in the direction of 11 pulsars. In the direction of three pulsars $(\mathrm{B} 0329+54$, $\mathrm{B} 0823+26$, and B1929+10), the spectrum power of spatial inhomogeneities of the interstellar plasma turned out to be very close to the value for the Kolmogorov spectrum $(n=3.67)$. For other pulsars, it ranges from 3.18 to 3.86 . It is shown that the measured scintillation parameters are significantly influenced by the duration of the observation session, expressed by its ratio to the characteristic scintillation time. If this parameter is less than 10, the parameter estimates may be biased: the values of $\alpha$ and the characteristic scintillation time $t_{\text {scint }}$ may decrease.
\end{abstract}

Keywords: pulsars, scintillations, interstellar plasma

DOI: $10.1134 / \mathrm{S} 1063772921120052$

\section{INTRODUCTION}

Inhomogeneities in the interstellar plasma scatter radio emission of space sources. Intense studies of scattering effects began with the discovery of pulsars, since pulsars are ultra-compact objects with coherent radiation and the influence of the source structure can be neglected. The scattering is manifested as image blurring, pulse broadening, intensity modulation over time and frequency (scintillation), and distortion of radio spectra. The parameters characterizing these phenomena are scattering angle $\theta_{\mathrm{sc}}$, scattering time $\tau_{\mathrm{sc}}$, characteristic scintillation time $t_{\mathrm{scint}}$, and characteristic decorrelation band $f_{\text {scint }}$. Theoretical studies of scattering effects have established certain relationships between their parameters (see, e.g., [1-3]). They must be compared with the parameters measured in radio astronomical observations of pulsars.

In the analysis of scintillations, it three modes of averaging of observed values are considered: snapshot mode, averaging mode, and ensemble averaging mode. First, snapshot mode corresponds to the averaging time $T_{\text {obs }} \ll t_{\text {scint }}$, i.e., the averaging time is much shorter than the characteristic scintillation time. Second, analysis in the averaging mode corresponds to the opposite case, $T_{\mathrm{obs}}>t_{\text {scint }}$. Here, as scintillation time, we mean the diffraction scintillations time, which in most cases ranges from a few seconds to tens of minutes at meter and decimeter wavelengths. Third, there is also refractive scintillation with a characteristic time of several weeks and months [4]. Analysis of the observed parameters over such time intervals is named ensemble averaging [5]. In this study, we will analyze scintillations in the normal averaging mode, $T_{\text {obs }}>t_{\text {scint }}$.

We will use the observational data obtained during the implementation of the scientific program of the ground-space interferometer "Radioastron." These data have been used already in other studies [6-8]. Dynamic spectra $S(f, t)$ and two-dimensional correlation functions $C F(\Delta f, \Delta t)$ will be analyzed.

\section{OBSERVATIONS}

Pulsar studies were an important part of the scientific program of the "Radioastron" ground-space 
Table 1. The list of studied pulsars

\begin{tabular}{|c|c|c|c|c|c|c|c|c|}
\hline PSR & $T, \min$ & $\Delta t, \mathrm{~s}$ & $N_{t}$ & $T_{\text {obs }}, \mathrm{s}$ & $N_{f}$ & RT & Date & Code \\
\hline B0329+54 & 60 & 7.145 & 504 & 3600 & 4096 & GB & 26.11.2012 & raes $10 \mathrm{a}$ \\
\hline B0809+74 & 180 & 10.33 & 1045 & 10800 & 4096 & GB & 17.12.2012 & raes06g \\
\hline B $0823+26$ & 150 & 0.531 & 16950 & 9000 & 2048 & AR & 11.03 .2015 & rags04aj \\
\hline B0834+06 & 55 & 1.273 & 2600 & 3300 & 8192 & GB & 08.12 .2014 & rags04ah \\
\hline B0919+06 & 90 & 0.430 & 12558 & 5400 & 2048 & AR & 10.05 .2018 & rags $29 p$ \\
\hline $\mathrm{B} 1133+16$ & 120 & 1.188 & 6000 & 7200 & 1024 & AR & 03.02 .2018 & rags $29 g$ \\
\hline B $1237+16$ & 100 & 1.382 & 4340 & 6000 & 512 & AR & 22.12.2017 & $\operatorname{rags} 28 \mathrm{c}$ \\
\hline B1749-28 & 250 & 5.625 & 2666 & 15000 & 192 & PA & 26.05 .2014 & raks02az \\
\hline B1929+10 & 100 & 0.226 & 26550 & 6000 & 512 & AR & 05.05 .2015 & rags04ap \\
\hline B1933+16 & 90 & 0.358 & 15083 & 5400 & 8192 & AR & 01.08.2013 & rags02aa \\
\hline B2016+28 & 45 & 0.558 & 5376 & 3000 & 2048 & AR & 22.05 .2015 & rags04aq \\
\hline
\end{tabular}

PSR - the pulsar name; $T$-total observations time, min; $\Delta t$-timestep, s; $N_{t}$-the number of sets in the spectrum; $T_{\mathrm{obs}}-$ the timespan of a single spectrum, s; $N_{f}$-the number of channels; RT - the telescope: GB-100-m Green-Bank radio telescope, AR-300-m Arecibo radio telescope, PA-64-m Parkes radio telescope; Date-the date of observations; Code-the code of the experiment.

interferometer. The observations were carried out mainly at the frequency of $324 \mathrm{MHz}$ and, in some cases, at the frequency of $1668 \mathrm{MHz}$. The receiver frequency bands were 16 and $32 \mathrm{MHz}$, respectively. These studies were mainly aimed at the properties and spatial distribution of interstellar plasma. The distances to effective scattering screens were determined by comparing the angular broadening $\theta_{\mathrm{sc}}$ with the characteristic time of the pulse scattering $\tau_{\mathrm{sc}}$ [6-9]. The pulsars selected for our study and their parameters are listed in Table 1. In this paper, we will use the dynamical spectra of pulsars $S(f, t)$ defined earlier for other purposes. The details concerning construction of dynamic spectra may be found in the publications cited above. The individual spectrum for the given time $t$ is calibrated as

$$
S(f, t)=\left[S^{\mathrm{ON}}(f, t)-S^{\mathrm{OFF}}(f, t)\right] / S^{\mathrm{OFF}}(f, t)
$$

where $S^{\mathrm{ON}}(f, t)$ and $S^{\mathrm{OFF}}(f, t)$ are the spectra obtained in time windows during the pulse of the pulsar and out the pulse, respectively. Each dynamic spectrum contains $N_{f} \times N_{t}$ values, where $N_{f}$ is the number of frequency channels and $N_{t}$ is the number of spectra in the given observational sample. Usually, the time interval between successive spectra is equal to the period of the pulsar, but in some cases, averaging over several periods was performed in order to smooth out the intrinsic variations in the intensity of the pulsar from pulse to pulse. All these parameters are listed in Table 1. In our list, two pulsars (B1749-28 and B1933+16) were observed at the frequency $1668 \mathrm{MHz}$ and the rest of objects-at the frequency $324 \mathrm{MHz}$.

\section{THE ANALYSIS METHOD OF DYNAMIC SPECTRA}

As it was already noted in the Introduction, for a justified assessment of the scattering parameters, we analyzed the dynamic spectra in the time interval $T_{\text {obs }}>t_{\text {scint }}$. For this purpose, two-dimensional correlation functions of dynamic spectra were calculated over the entire observation interval. The two-dimensional correlation function $C F(\Delta f, \Delta t)$ was calculated as

$$
\begin{gathered}
C F(\Delta f, \Delta t)=\left[\left(N_{f}-\Delta f\right)\left(N_{t}-\Delta t\right)\right]^{-1} \\
\times \sum_{\Delta f=0}^{N_{f}-\Delta f} \sum_{\Delta t=0}^{N_{t}-\Delta t} \Delta S(f, t) \Delta S(f+\Delta f, t+\Delta t),
\end{gathered}
$$

where $\Delta f$ and $\Delta t$ are frequency and time delays, while $\Delta S(f, t)=S(f, t)-\langle S(f, t)\rangle$, where

$$
\langle S(f, t)\rangle=\left[N_{f} N_{t}\right]^{-1} \sum_{i=0}^{N_{f}} \sum_{j=0}^{N_{t}} S\left(f_{i}, t_{j}\right) .
$$

Then, we used normalized correlation functions $N C F(\Delta f, \Delta t)=C F(\Delta f, \Delta t) / C F(0,0)$. Our task was to estimate the characteristic scintillation time $t_{\text {scint }}$. Following Cordes [5], we tried to determine the value of the scintillation time from the time-section of twodimensional correlation functions of dynamic spectra $C F(\Delta f, \Delta t)$ taken with zero frequency delay. Traditionally [5], this cross-section is approximated by a Gaussian and the half-width of this function at the level 1/e is taken as the scintillation time. Other researchers, who used correlation functions for data analysis [2, $10,11]$, have pointed out deviations of these functions from the Gaussian form. We suggest to use a more universal function for approximation of the time-sec- 
tion $N C F$, namely, an exponential function with an arbitrary power:

$$
Y(\Delta t)=A \exp \left(-(\Delta t)^{\alpha} / B\right) .
$$

Here, $A$-amplitude, $B$-the parameter characterizing the width of $N C F$, the half-width at the level 1/e is defined as $W_{e}=t_{\text {scint }}=B^{1 / \alpha}$. The parameters $A, \alpha, B$ were determined by approximation of computed $N C F$ by function (4) for positive shifts with the step of 2 counts from $\Delta t=0$ made for exclusion of noise peak.

\section{ANALYSIS RESULTS}

Analysis results are presented in Table 2. The first column of the table gives the designation of the pulsar, the second one - the ratio of the time at which the dynamic spectrum was obtained $\left(T_{\text {obs }}\right)$ and characteristic scintillation time $\left(t_{\text {scint }}\right)$, and the third one shows the characteristic scintillation time $\left(t_{\text {scint }}\right)$ determined by the relation $W_{e}=B^{1 / \alpha}$. The next two columns of the Table compare the values of the root mean square deviations (RMS) for two options for approximation of the calculated $N C F$ ones: by the traditional Gaussian and by the exponent with an arbitrary power; the last column shows this power. The numbers in the parentheses give the value of the formal RMS error of determination of this parameter as a result of the least squares fit. These errors refer to the last significant digits of the measured values. In fact, these parameters change over time in a much wider range of values.

Cordes et al. [12] called attention to the so-called "relative error" in their estimates of the decorrelation band, which arises in the analysis of correlation functions. They estimated the relative error by the value $1 / \sqrt{N}$, where $N$ is the total number of scintillation details (scintiles) in the spectrum $N=\frac{T_{\text {obs }} B_{f}}{\Delta v_{i s s} t_{\text {scint }}}$ and $B_{f}$ is the bandwidth of the receiver. This error, when $\Delta v_{i s s}$ is determined from the CCF, usually is $10-20 \%$. We call attention to the fact that in determination of $t_{\text {scint }}$, along with random errors as the indicated relative error, a systematic error also appears, which is also associated with restrictions on the observation time and the receiver bandwidth. This error arises due to the cutting of individual scintiles, which is due to the limited duration of the observational scan in time and frequency $\left(T_{\text {obs }}, B_{f}\right)$. We suggest to introduce a "cutting parameter" $R$, which is related to the number of scintiles truncated by the edges of the observation field in time. As such a truncation parameter, we will use the ratio value of the duration of the observational scan and the value of the characteristic scintillation time $R=T_{\text {obs }} / t_{\text {scint }}$; the values of $R$ are given in column 2 of Table 2. To compare the quality of the
Table 2. Results

\begin{tabular}{c|r|c|c|c|l}
\hline PSR & \multicolumn{1}{c|}{$R$} & \multicolumn{1}{c}{$t_{\text {scint }}, \mathrm{s}$} & $\mathrm{RMS}_{1}$ & $\mathrm{RMS}_{2}$ & $\alpha$ \\
\hline $\mathrm{B} 0329+54$ & 31.3 & $115(5)$ & 1.62 & 0.58 & $1.67(1)$ \\
$\mathrm{B} 0809+74$ & 24.5 & $373(50)$ & 0.90 & 0.22 & $1.33(1)$ \\
$\mathrm{B} 0823+26$ & 212.0 & $50(3)$ & 0.06 & 0.04 & $1.66(1)$ \\
$\mathrm{B} 0834+06$ & 14.5 & $227(5)$ & 0.86 & 0.25 & $1.528(6)$ \\
$\mathrm{B} 0919+06$ & 55.3 & $94(2)$ & 0.08 & 0.03 & $1.57(1)$ \\
$\mathrm{B} 1133+16$ & 263.1 & $26.6(3)$ & 0.40 & 0.13 & $1.86(1)$ \\
$\mathrm{B} 1237+16$ & 22.1 & $221(15)$ & 1.27 & 0.34 & $1.39(1)$ \\
$\mathrm{B} 1749-28$ & 68.6 & $201(8)$ & 0.57 & 0.32 & $1.82(1)$ \\
$\mathrm{B} 1929+10$ & 25.9 & $232(5)$ & 0.73 & 0.24 & $1.65(2)$ \\
$\mathrm{B} 1933+16$ & 128.6 & $42(8)$ & 2.63 & 0.67 & $1.18(1)$ \\
$\mathrm{B} 2016+28$ & 4.2 & $708(70)$ & 1.40 & 0.16 & $1.36(2)$ \\
\hline
\end{tabular}

PSR-the pulsar name; $R=T_{\text {obs }} / t_{\text {scint }} ; t_{\text {scint }}$-scintillations time, s; $\mathrm{RMS}_{1}$ and $\mathrm{RMS}_{2}-\mathrm{RMS}$ for Gaussian and for exponential functions, respectively; $\alpha$-the power for exponential function.

approximations of $N C F$ calculated by simple Gaussians and by exponents with an arbitrary power, we show the residual deviations for these cases in Table 2. It is seen that these residual deviations are always noticeably smaller (from 1.5 to 8.8 times) for the approximations by exponents with an arbitrary power. Figure 1 shows, as an example of comparison of these methods of approximation, the data for two pulsars: $\mathrm{B} 1237+25$ and $\mathrm{B} 2016+28$. In the upper part of the figure, different lines show the calculated $N C F$ and two approximation functions. In the scale of such a figure, it is difficult to discern the difference between the approximations. For this reason, in the lower part of the Figure, deviations of the observational data from the approximation functions are shown: the dark (solid) line corresponds to the approximation by exponents with an arbitrary power and the lighter (dashed) line corresponds to the approximation by Gaussians. It is clearly seen that approximation by exponents with an arbitrary power reflects the observed $N C F$ much better.

\section{DISCUSSION}

Interstellar scattering is closely related to the properties of the interstellar scattering medium (ISM), since the scintillation effects are caused by fluctuations of the electron density in the latter. An analysis of a large amount of observational data on pulsars has shown that pulsar scintillations are statistically consistent with the power-law spectrum of spatial inhomogeneities with an index $n=3.67$ (i.e., the Kolmogorov spectrum) in a very wide range of spatial scales [13, 14]. The structure function of oscillations of the phase of pulsars radio emission is related to the spatial spectrum of the electron density, which is described by the 

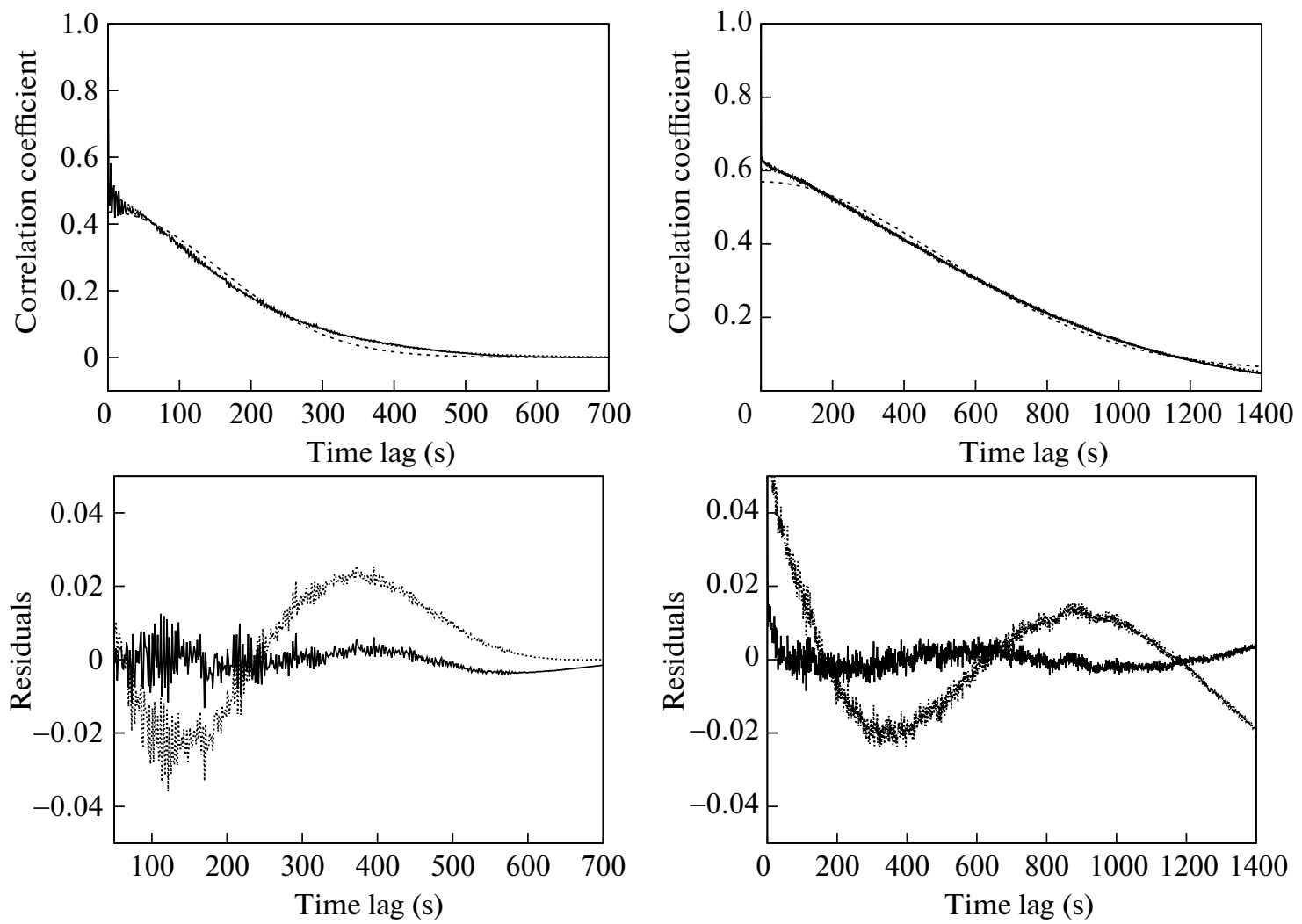

Fig. 1. Upper panel-examples of time cross-sections for two-dimensional autocorrelation functions of the dynamic spectra of pulsars B1237+25 (left) and B2016+28 (right). Solid lines show the calculated functions, dashed lines represent the approximation exponential functions, and the dotted lines are the Gaussians. The bottom panel shows the corresponding residual deviations: solid lines represent the results of approximation by power functions, and dashed lines-by Gaussians.

relation $\Phi(q)=C q^{-n}$, where $q$ is the spatial frequency and $C$ characterizes the degree of turbulence.

The analysis of the structure functions of some pulsars has shown that the spectrum for different local directions in the Galaxy may differ from the Kolmogorov spectrum $[8,15]$. The relationship between the index of the spectrum of inhomogeneity $n$ and the power in the time dependence of the structure function $\alpha$ was discussed in several publications $[3,16,17]$. In the paper by Shishov et al. [3], it was shown that, for the power-law spectrum in the regime of saturated scintillations, the temporal structure function $D(\Delta t)$ has the form $D(\Delta t)=\left(\Delta t / t_{\text {scint }}\right)^{\alpha}$ for $\Delta t \ll t_{\text {scint }}$ and $\alpha=n-2$. The structure function $D(\Delta t)$ can be expressed in terms of the normalized correlation function $N C F$ as $D(\Delta t)=2(N C F(0)-N C F(\Delta t))$. For the case of approximating the correlation function by an exponent, we obtain the expression for small delays:

$D(\Delta t)=2\left(N C F(0)-N C F(\Delta t)=(\Delta t)^{\alpha} / B\right.$. Thus, for small delays, the structure function is a power-law with an index $\alpha$ and the spectrum power of spatial inhomogeneities of the interstellar plasma in this approximation will be $n=\alpha+2$. The powers $\alpha$ are shown in the last column of Table 2. The numbers in parentheses correspond to the curve negotiation errors of the power function.

It turned out that in the direction of three pulsars (B0329+54, B0823+26, and B1929+10) out of 11 pulsars in our list, the spectrum exponent of spatial inhomogeneities of the interstellar plasma $n$ is very close to the value of the Kolmogorov spectrum $(n=3.67)$. The highest value $n=3.86$ was obtained in the direction to the pulsar B1133+16 and the smallest value $(n=3.18)-$ in the direction to the pulsar B1933+16. It should be noted that the correct approximation of the correlation function (power-law) gives the correct value of the power $n$. It can also be noted that the difference between the correlation function and the Gaussian shows that the internal scale of turbulence is smaller than the characteristic spatial scale of scintillations.

As indicated in the previous paragraph, a systematic error in the determination of the characteristic scintillation time $t_{\text {scint }}$ and in the determination of the indicator can be introduced by the effect of the limited observational time at which the dynamic spectra. We propose to characterize this effect by the parameter 


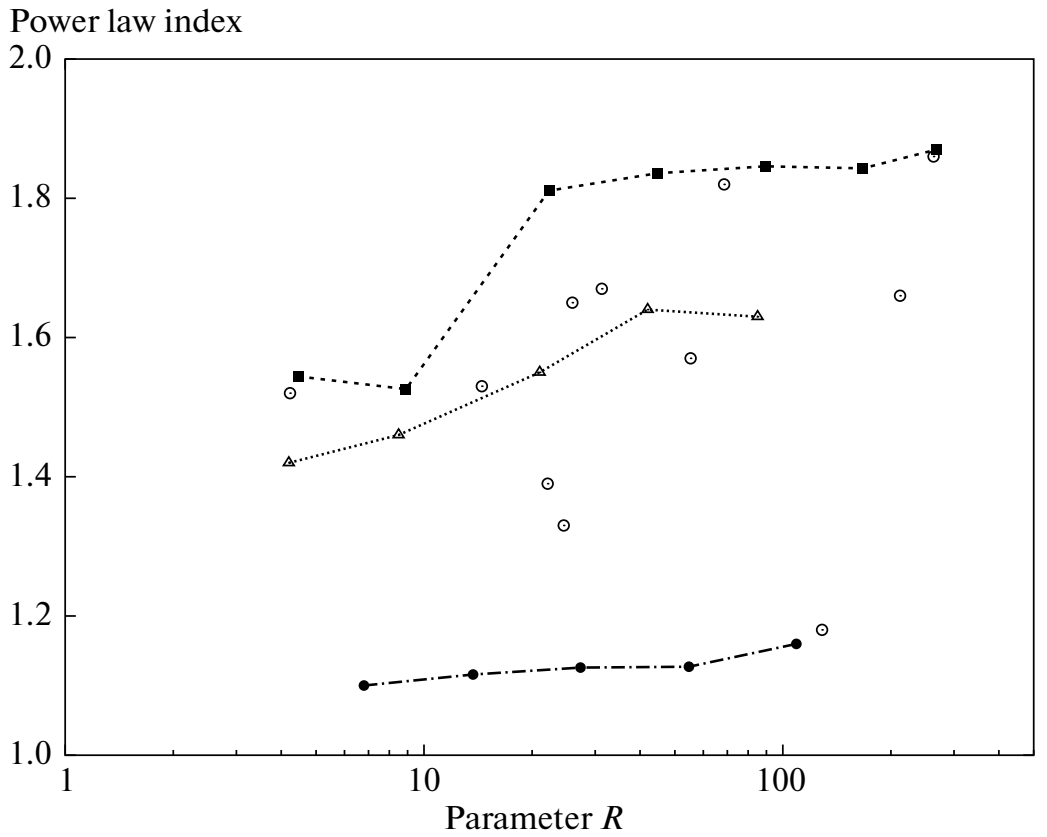

Fig. 2. Dependence of the index of the power-law approximation function $\alpha$ on $R$-the ratio of the observation time $T_{\text {obs }}$ to the characteristic scintillation time $t_{\text {scint }}$. White circles show the measured values for 11 pulsars. The dashed line passing through the squares reflects the behavior of $\alpha$ for the pulsar B1133+16 with an artificial shortening of the observation time. The dotted line passing through triangles shows the same for the pulsar B $0823+26$, and the dash-dotted line with black dots refers to the pulsar B1933+16.

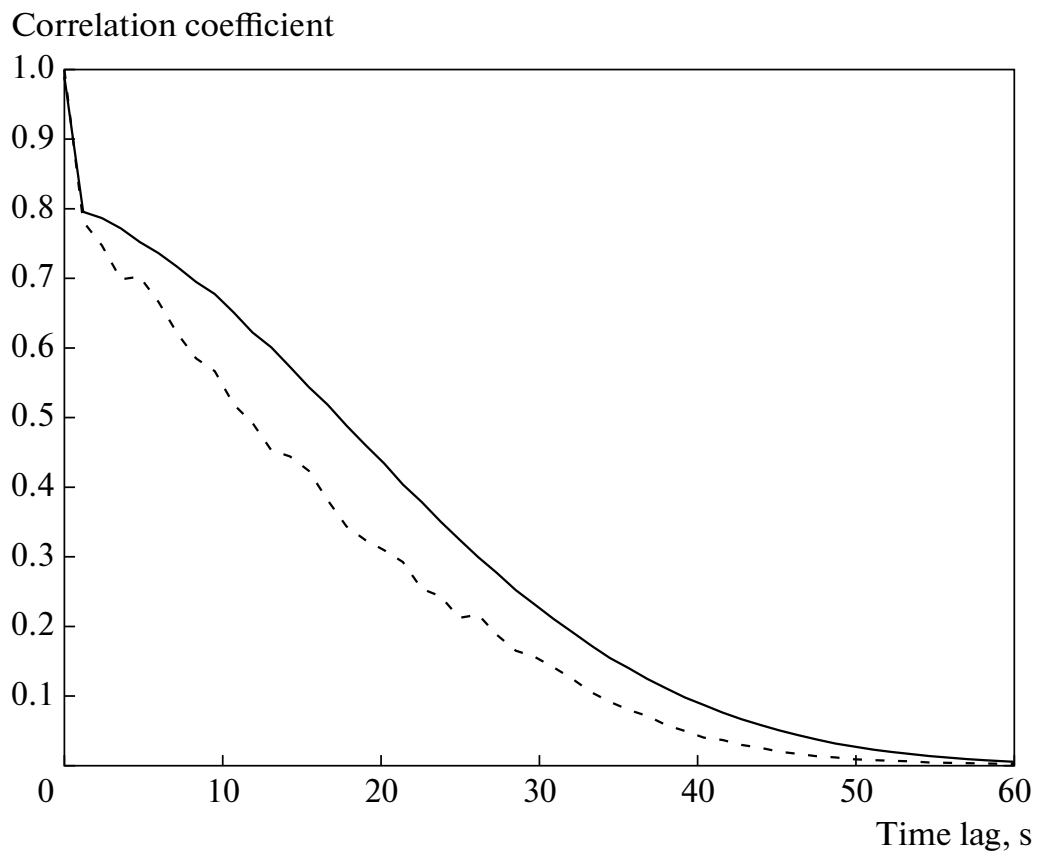

Fig. 3. Comparison of the cross-sections in time for two-dimensional autocorrelation functions of the dynamic spectrum of the pulsar B1133 + 16 for the total observational time of $7000 \mathrm{~s}$ (solid line) and for a limited observation interval of $100 \mathrm{~s}$ (dashed line).

$R=T_{\text {obs }} / t_{\text {scint }} ; R$ values are given in column 2 of Table 2. We will discuss this influence based on the data presented in Fig. 2. In this figure, the abscissa scale is given in a logarithmic scale, and the ordinate scale is left in natural values. Circles indicate the val- ues of the exponent $\alpha$ obtained by the method of approximating the correlation function. For the main group of points, you can see some tendency of an increase in the exponent $\alpha$ with an increase in the parameter $R$. To check this tendency, for three pulsars 
of which the initial value of $Z$ exceeds 100 , we carried out processing with artificial limitation of the observation time. The dashed line through the squares refers to the pulsar B1133+16 (top line). For this pulsar, the total number of pulses is $N=6000$, and we performed data processing for the values of $N$ equal to 6000 , $4000,2000,1000,500,200$, and 100 . It can be seen from the figure that at small values of the parameter $R$, a noticeable decrease in the values of the indicator is observed. The dotted line through the triangles refers to the pulsar B0823+26. Decreasing trend of $\alpha$ with a decrease in the observation time is confirmed for this pulsar as well. This trend is also seen for the pulsar B1933+16 (dash-dotted line passing through black circles), although for this pulsar the indicator $\alpha$ has the lowest value out of total groups of points in the figure. This pulsar has the greatest value of the measure of dispersion $\left(D M=158 \mathrm{kpc} / \mathrm{cm}^{3}\right)$ and is located at a considerable distance $(3.7 \mathrm{kpc})$. We also note that the RMS for the Gaussian in relation to the RMS for the power function increases with decreasing parameter $R$.

The duration of the observation session also affects the determination of the characteristic scintillation time $t_{\text {scint }}$. Figure 3 shows a comparison of the $N C F$ time cross sections for the full (7000 s) and truncated (100 s) observational scans: it can be seen that with a decrease in $R$, the cross section shape changes and $t_{\text {scint }}$ decreases. The smaller the $R$, the greater the effect is. Apparently, the reason for the dependence of the estimates of the exponent and the characteristic scintillation time on the relative sample length is the insufficient number of diffraction spots in the analyzed dynamic spectrum to obtain a statistically correct estimate of the parameters. With a small $T_{\text {obs }} / t_{\text {scint }}$ ratio, some of the diffraction structures are cut off both in time and in frequency if $f_{\text {dif }}$ is only several times smaller than the receiver bandwidth.

\section{CONCLUSIONS}

1. We have carried out the analysis of normalized two-dimensional correlation functions $N C F$ of dynamic spectra for 11 pulsars using the archival data of the "Radioastron" project. The time-sections of these functions were approximated by exponential functions with a power $\alpha$. It was shown that these functions describe the shape of NCF much better than the Gaussian functions. For all sources, the power $\alpha$ and characteristic scintillation time $t_{\text {scint }}$ were determined.

2. Out of 11 pulsars in our list, in the direction of three pulsars (B0329+54, B0823+26, and B1929+10), the spectrum power of spatial inhomogeneities of the interstellar plasma turned out to be very close to the value for the Kolmogorov spectrum $(n=3.67)$. For other pulsars, it ranges from 3.18 (PSR B1933+16) to 3.86 (PSR B1133+16). The average value of this power for the entire list, with the exception of the pulsars B2016+28 and B1933+16, is 3.62.

3 . It was shown that the duration of the observation session, expressed in the units of the characteristic scintillation time (parameter $R$ ), has a noticeable effect on the measured parameters. If this parameter is less than 10, biased estimates of the parameters can be obtained: a decrease in the values of the power $\alpha$ and the characteristic scintillation time $t_{\text {scint }}$. Out of our list, the pulsar B2016+28 definitely belongs to this category.

\section{ACKNOWLEDGMENTS}

The project "Radioastron" was carried out by the AstroSpace Center of the P.N. Lebedev Physics Institute of the Russian Academy of Sciences and S.A. Lavochkin Research and Production Association under a contract with the State Corporation ROSCOSMOS together with many scientific and technical organizations in Russia and other countries.

In this publication, we used the results of pulsars observations carried out for the "Radioastron" program with the following radio telescopes: $100-\mathrm{m}$ radio telescope of the Green Bank Observatory, which is an institution of the National Science Foundation (NSF) and is operated in accordance with a cooperation agreement with the Association of Universities (Associated Universities, Inc.); the Arecibo Observatory $300-\mathrm{m}$ radio telescope which was operated by SRI International under a collaborative agreement with the National Science Foundation (AST1100968) and in alliance with Ana G. Mendes University (Metropolitana University and the University Space Research Association); as well as the 64-m Parkes Radio Telescope is a part of the Australian Telescope (ATNF), which is funded by the Australian government as a national facility operated by CSIRO.

We acknowledge E.N. Fadeev (ASC of the Physical Institute) for providing dynamic spectra from the personal archive and useful advice during preparation of the paper.

\section{OPEN ACCESS}

This article is licensed under a Creative Commons Attribution 4.0 International License, which permits use, sharing, adaptation, distribution and reproduction in any medium or format, as long as you give appropriate credit to the original author(s) and the source, provide a link to the Creative Commons licence, and indicate if changes were made. The images or other third party material in this article are included in the article's Creative Commons licence, unless indicated otherwise in a credit line to the material. If material is not included in the article's Creative Commons licence and your intended use is not permitted by statutory regulation or exceeds the permitted use, you will need to obtain permission directly from the copyright holder. To view a copy of this licence, visit http://creativecommons.org/licenses/by/4.0/. 


\section{REFERENCES}

1. B. J. Rickett, Ann. Rev. Astron. Astrophys. 15, 479 (1977).

2. V. I. Shishov, Sov. Astron. 17, 598 (1974).

3. V. I. Shishov, T. V. Smirnova, W. Sieber, V. M. Malofeev, et al., Astron. Astrophys. 404, 557 (2003).

4. D. R. Stinebring, V. I. Smirnova, T. H. Hankins, J. S. Hovis, V. M. Kaspi, J. C. Kempner, E. Myers, and D. J. Nice, Astrophys. J. 539, 300 (2000).

5. J. M. Cordes, Astrophys. J. 311, 183 (1986).

6. M. V. Popov, N. Bartel, C. R. Gwinn, M. D. Johnson, et al., Mon. Not. R. Astron. Soc. 465, 978 (2017).

7. C. R. Gwinn, M. V. Popov, N. Bartel, A. S. Andrianov, et al., Astrophys. J. 822, 96 (2016).

8. E. N. Fadeev, A. S. Andrianov, M. S. Burgin, M. V. Popov, A. G. Rudnitskiy, V. I. Shishov, T. V. Smirnova, and V. A. Zuga, Mon. Not. R. Astron. Soc. 480, 4199 (2018).

9. M. V. Popov, N. Bartel, M. S. Burgin, C. R. Gwinn, T. V. Smirnova, and V. A. Soglasnov, Astrophys. J. 888, 57 (2020).
10. J. M. Armstrong and B. J. Rickett, Mon. Not. R. Astron. Soc. 194, 623 (1981).

11. A. Wolszczan, Mon. Not. R. Astron. Soc. 204, 591 (1983).

12. J. M. Cordes, J. M. Weisberg, and V. Boriakoff, Astrophys. J. 288, 221 (1985).

13. J. W. Armstrong, B. J. Rickett, and S. R. Spangler, Astrophys. J. 443, 209 (1995).

14. V. I. Shishov and T. V. Smirnova, Astron. Rep. 46, 731 (2002).

15. T. V. Smirnova, V. I. Shishov, M. V. Popov, C. R. Gwinn, et al., Astrophys. J. 786, 115 (2014).

16. T. V. Smirnova and V. I. Shishov, Astron. Rep. 52, 736 (2008).

17. A. S. Andrianov, T. V. Smirnova, V. I. Shishov, C. R. Gwinn, and M. V. Popov, Astron. Rep. 61, 513 (2017).

Translated by L. Yungelson 